\title{
Sorrow never leaves us where it found us
}

Sorrow's Reward

Robert Allan Bear

Kingsley Publishing; 2011

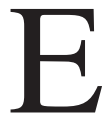
arly in his medical training Robert Allan Bear came upon a copy of Edgar Lee Masters' Spoon River Anthology. That book, published in 1915, is a work of poetic fiction about the inhabitants of the small American town of Spoon River, all now interred in the local cemetery. Each person in turn declares the fragmented, intertwining truths and half-truths of their lives.

A hundred years later, Bear, a retired nephrologist, adopts a similar literary structure to examine the microcosm of the dialysis unit and the various members of that "community." Patients, family

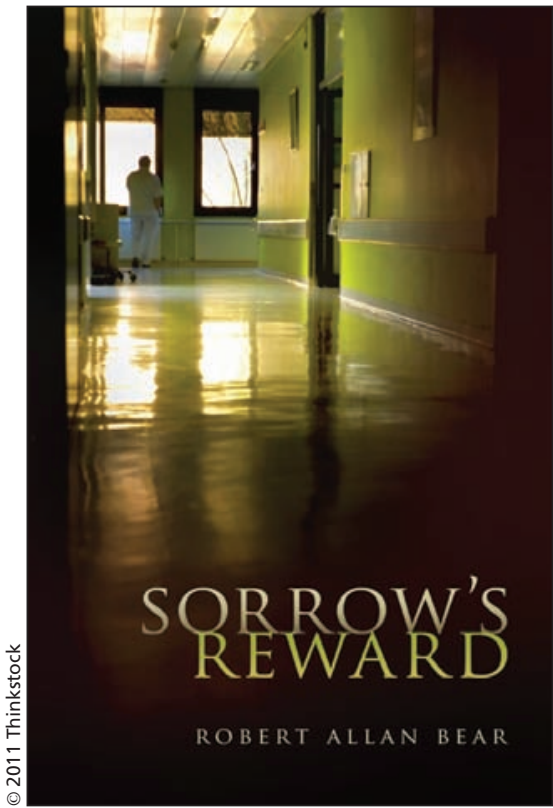

The ironic reality of loss, diminishment and disorientation in each character's life is captured in the book's title, and its epigraph: "Sorrow has its reward. It never leaves us where it found us." Sorrow also has its punishment. In the crucible of the dialysis unit we are all changed — but most of us for worse rather than for better.

The novel is two stylistically different stories combined into one book. Most of the book is written in "Spoon River" epitaph style - various characters address short poetic statements directly to the reader.

The other story is the doctor-patient relationship of main characters, David Walk and Carole D'Amico. They are the only two characters in the book who have conversation directly with each other, mostly in the form of lengthy dialogues of questions and answers about the nature of health care as it plays out in the unit. members, doctors, nurses, trainees, clerks, hospital administrators and technicians speak in first person poetic vignettes revealing piecemeal the meaning of work and illness, love and death. Sometimes the meaning discovered is the apparent absence of meaning.

Early on in the book, Carole D'Amico, a patient, and a self-described "academic, and expert in organizational culture," gives her impressions of the unit. To describe her fellow patients she chooses words like "decrepitude, resignation, fear, intransigence, anger, self-absorption, throbbing sexuality." For the staff the descriptors are "weariness, discontent, ennui and the rarity of laughter." The dialysis unit is not a happy place.

Loss is a major theme in the lives of both the individuals afflicted by chronic kidney disease and the health workers caring for them. In the following vignette, a drum beat of lose, loss and lost sounds in each line:

\section{Dr. David Walk Reflects upon a Patient "Lost"}

All of us in the Unit lost Ollie.

Peter Lossing told me he lost a patient last week.

I saw a man in my office yesterday; he told me he had

lost his wife and

daughter in an accident.

(He also told me he had lost all interest in his disease and its treatment;

that without them, he was just life lost.)

We all know what it means to lose some one.

The word lose doesn't capture it though, does it?

It doesn't even come close.
Their relationship is partly driven by loneliness and lessons learned from past failed marriages. They also share a passion for what Carole refers to as "the culture and ecology of the place," for what should be the dialysis unit's "core values." Dr. Walk is disillusioned by the fruitlessness of his past efforts to address the quality issues; she, meanwhile, is attracted to him and energized by observations of the system from her new vantage point of patient. Perhaps they will build something better together.

Theirs is an interesting story by itself and deserves to be expanded its own venue - perhaps in a different book. In the shifting back and forth between the realism of their conversation and the stylized pronouncements of the other characters, this reader became increasingly aware of the stylistic dissonance. The story's momentum begins to drift away from the larger cast of less-developed characters to the intellectual and romantic preoccupations of one doctor and one patient.

In many respects, the two stories tell the same story. The difficulty is that even though the stories are related, they eventually get in each other's way. The double narrative structure undermines the book's overall effect.

That weakness aside, Bear's book is a good addition to the collection of contemporary writings by physicians meditating on their work, especially in the arena of chronic care. The voices in Sorrow's Reward, and the cautionary tale they tell, speak to all patients and health care workers today.

\section{Vincent Hanlon MD \\ Family physician \\ Lethbridge, Alta.}

CMAJ 2012. DOI:10.1503/cmaj.111368 